\title{
Abandon et prise en charge des enfants en Afrique : une problématique centrale pour la protection de l'enfant
}

\author{
Valérie DELAUNAY ${ }^{1}$
}

\begin{abstract}
T 'enfant et le respect de ses droits sont au cœur de nombreux débats. Enfants vulnérables, négligés, victimes, exploités, "trafiqués", abusés sexuellement, sont autant de groupes identifiés comme cibles par les programmes de protection de l'enfance.

Actuellement, les savoirs sur l'enfance, particulièrement en Afrique, sont fortement orientés par la vision de l'enfance véhiculée par les grandes organisations internationales (Programme des Nations unies pour le développement, Organisation mondiale de la santé, Banque mondiale, UNICEF), qui se fonde sur le concept de "protection de l'enfance". L'enfant est vulnérable, il subit différentes formes de violences, maltraitances, négligences, exploitation et les États se doivent de les protéger. Ceux-ci s'engagent à travers la ratification de textes internationaux tels que la Convention des droits de l'enfant (1989) à mettre en place une réelle politique de protection de l'enfance. Des efforts sont menés par les organisations internationales pour produire des indicateurs mesurant le niveau de vulnérabilité et visant à évaluer les progrès accomplis (ODEROI, 2006 ; UNICEF, 2002, 2007a, 2007b). Les résultats sont quantitatifs et renseignent sur l'évolution de la survie de l'enfant (niveaux de mortalité, causes probables de décès, malnutrition, vaccination), sur son éducation (taux de scolarisation).

Si les progrès sont considérables en termes de recherche sur la protection de l'enfance dans les pays du Nord (Leventhal, 2003), force est de constater les lacunes en termes de descriptions, de définitions et de compréhension des différents aspects touchant à la protection de l'enfant en Afrique (Lachman, 2004). En effet, de nombreux aspects demeurent ignorés. Les situations de violence, négligence, maltraitance, abandon, les conditions de prise en charge, y compris des orphelins, et les réponses institutionnelles mises en place, sont très peu documentées. Les difficultés de l'étude de ces phénomènes sont en partie liées au fait qu'en Afrique la perception de la maltraitance envers les enfants

1 Chargée de recherche à l'Institut de recherche pour le développement, Laboratoire Population-Environnement-Développement, UMR151 Université de Provence/IRD, IRD BP434 Antananarivo, Madagascar, Valerie.Delaunay@ird.fr
\end{abstract}


demeure du domaine de la sphère privée et familiale (Lachman, 1996). La violence sexuelle envers les enfants n'est abordée que dans certains pays (surtout en Afrique du Sud) et auprès de groupes spécifiques (universitaires ou cas cliniques) (Lalor, 2004). Les données produites restent parcellaires et anecdotiques, utilisées de manière illustrative pour justifier les recommandations en matière de mise en place de politique de protection.

Face au manque de données, la vision des organisations internationales prend donc appui sur une approche a priori qui permet seulement de constater la complexité des situations et des comportements à travers les sociétés et les cultures comme au sein d'un même groupe. Le savoir, anecdotique ou hypothétique, est utilisé comme bon ou mauvais exemple relativement à une certaine éthique ou morale, accusant ou valorisant des acteurs et des pratiques et visant à protéger l'enfant (contre qui ? contre quoi ?), jugé vulnérable.

Comment la recherche peut-elle alors contribuer à élaborer des éléments nécessaires pour une réflexion a posteriori sur la protection de l'enfance ? À la lecture et à l'analyse des différents travaux de sciences sociales disponibles, une problématique apparait centrale dans le concept de protection de l'enfance : il s'agit de sa prise en charge. Qu'elle soit familiale, extra-familiale ou institutionnelle, ce sont effectivement les ruptures de cette prise en charge qui conduisent l'enfant à des situations d'exploitation, de maltraitance, de négligence ou d'abandon. Les problèmes rencontrés par les enfants à travers le monde, qu'il s'agisse de la pauvreté, la guerre, la dislocation familiale, la violence familiale, l'abandon, le VIH/sida, se cristallisent autour de leur prise en charge. À qui revient la prise en charge de l'enfant à sa naissance ? Qu'advient-il lors de situation de crise, lorsque les tuteurs naturels ne sont plus en mesure de prendre l'enfant en charge? Quelles solutions offrent le milieu familial? Quelles solutions sont proposées par la société civile ? Par l'État?

La sociologie et l'anthropologie de l'enfance ont permis de mettre en évidence la pluralité de l'enfance et ses fondements sociaux variant à travers le monde. Dans un article récent, Bonnet et de Suremain $(2008,16)$ rappellent que, en Afrique notamment, la mère est rarement l'unique responsable du développement de l'enfant et que sa prise en charge est habituellement partagée. L'enfant n'est pas l'enfant de l'individu ou du couple, mais celui du lignage, de la "famille élargie" ou encore de la "grande famille", selon les expressions consacrées. Des systèmes de circulation, au sein de la parenté ou de l'entourage proche, facilitent la prise en charge des enfants que les tuteurs immédiats, généralement le père et la mère biologiques, ne parviennent pas toujours à assurer lors des situations de crise (séparation, décès, maladie, perte d'emploi, famille trop nombreuse).

La question de la prise en charge de l'enfant et de son abandon, prend dès lors place dans un contexte social et culturel caractérisé à la fois par des systèmes de représentations et de normes dans lesquels les comportements de rejet, y compris extrêmes (infanticide), prennent leur signification et par des pratiques de circulation et de confiage des enfants, ou fosterage selon la terminologie adoptée par les anthropologues (Lallemand, 1993). Les pratiques d'abandon font partie 
intégrante de cet ensemble de comportements et de croyances qui leur donne sens. Il est clair que l'abandon ne peut être compris que dans son contexte culturel, social et historique particulier (Panter-Brick \& Smith, 2000). L'abandon prend diverses formes qui peuvent conduire au décès de l'enfant (pratiques d'infanticide), à grossir le nombre des enfants des rues (pratiques de négligence, de maltraitance), des enfants domestiques (pratiques d'exploitation), tout comme il peut se rapprocher d'un confiage, temporaire ou définitif, dans l'intérêt de l'enfant (pratiques de confiage ou de don).

L'objectif de cet article est de faire le bilan de la connaissance, à travers la littérature disponible, de la question de l'abandon et de la prise en charge de l'enfant dans le contexte africain, de décrire les différentes situations existantes et de contribuer à l'amélioration des définitions et des savoirs. Nous observerons comment les fondements culturels et sociaux de l'abandon d'enfant aident à comprendre les pratiques de rejet et conduisent à s'interroger sur leur disparition et/ou leurs transformations actuelles. Nous verrons aussi comment les systèmes harmonieux de circulation des enfants répondent aux situations de crise familiale dans la prise en charge des enfants mais présentent actuellement des formes perverties dont les conséquences sont au détriment des enfants. Nous discuterons, enfin, comment le concept de prise en charge de l'enfant, central en matière de protection de l'enfance, peut être appréhendé par l'analyse de données existantes et par des approches de terrain appropriées.

\section{LES FONDEMENTS CULTURELS ET SOCIAUX DE L'ABANDON}

\subsection{L'infanticide : des pratiques abandonnées?}

Les systèmes de représentations du processus de procréation confèrent couramment à un esprit ou à une divinité le rôle d'insuffler la vie, le souffle pour donner au nouveau-né le statut d'être social à part entière. La cérémonie du nom est souvent le marqueur de ce passage et intervient dans un certain délai après la naissance. Certaines naissances "anormales" (enfants malformés, jumeaux, naissances par les pieds...) font l'objet d'infanticide, généralement de manière immédiate, alors que l'enfant est aux portes de la vie. Cet acte n'est pas assimilé à un meurtre, d'autant que le véritable être reviendra dans un autre corps: Le faire mourir n'est pas le supprimer, mais simplement l'ajourner. Il demeure un candidat immédiat à la vie prochaine (Erny, 1988). Ces enfants ou naissances anormales sont interprétées comme un signe néfaste, une malédiction, un acte de sorcellerie, dont le préjudice pour l'entourage ne laisse d'autre issue que la mort de l'enfant ou son abandon par exposition. La survie de la mère, des parents, voire du clan en dépend. Ces pratiques répondent à des obligations sociales mettant en jeu l'ensemble du groupe et leur justification est celle d'un ordre (tant culturel que naturel) qui doit faire face au désordre (Singleton, 2004). 
Les travaux ethnologiques ont décrit de nombreuses pratiques d'infanticide ou d'abandon. Ainsi, certains enfants sont soupçonnés d'être des revenants : ceux qui naissent pour mourir (Lolo, 1991). Dans les sociétés traditionnelles du Sud Cameroun, les nouveaux-nés affligés d'une pathologie organique importante étaient voués à la mort, et parmi eux, des enfants autistes (Lolo, 1991, cité par Ezembé, 2003). Au Bénin, les enfants malformés, les nouveaux-nés issus d'un accouchement ayant causé la mort de la mère, ou d'un accouchement "anormal" (présentation dystocique) étaient victimes d'infanticide (Agossou, 2003 ; Mattern, 2007). Chez les Chaga du nord de la Tanzanie, l'infanticide était pratiqué en cas de naissances conçues hors mariage, de naissances anormales, de nouveaux-nés malformés, de jumeaux (Raum, 1967 ; Singleton, 2004). Au Ghana, les enfants malformés, ayant des dents, ou même nés après une grossesse difficile, sont suspectés d'être un esprit. Si l'esprit est découvert, il ne peut pas rester au monde. L'enfant doit absorber une potion toxique ; s'il y survit, on le pose dans la brousse pendant deux nuits; si toutefois il survit à ces deux nuits, il pourra reprendre sa place (Allotey \& Reidpath, 2001).

Les jumeaux font fréquemment l'objet de traitement particulier. Leur arrivée peut être interprétée positivement, comme chez les Kedjom du Cameroun (Diduk, 1993, 2001). Mais ils ont plus généralement été l'objet de fortes discriminations (Ball \& Hill, 1996). Au sud-est du Nigeria, dans l'aire culturelle Igbo, les jumeaux, considérés comme une abomination envers la divinité de la terre, étaient systématiquement éliminés (Achebe, 1967). Chez les M’bali d'Angola, la naissance de jumeaux représentait une calamité pour tout le pays, c'est pourquoi on les tuait (Erny, 1988). Chez les Antambahoaka du sud-est de Madagascar, ils étaient éliminés par l'intervention d'un sorcier pour protéger leurs parents (Van Gennep, 1904). La plupart de ces études relatent des observations de terrains relativement anciennes. Renne et Bastian (2001) relèvent le fait que les jumeaux ont attiré l'attention des ethnologues dans les années 1920, puis à la fin des années 1960 et dans les années 1970, mais que les études récentes sont rares.

Dans une réflexion visant à réévaluer l'infanticide des jumeaux, Ball et Hill (1996) s'interrogent sur la validité à le considérer comme un phénomène culturel. Les auteurs proposent de faire entrer l'élimination des jumeaux dans une catégorie qui justifie l'infanticide. Selon Renne et Bastian (2001, citant Schapera, 1927, 135), les pratiques relatives aux naissances gémellaires doivent être considérées comme une variation de celles relatives aux naissances simples. Il y a des catégories d'enfants qui justifient l'infanticide. C'est parce que les jumeaux entrent dans ces catégories qu'ils sont sujets à ces pratiques.

On suppose aujourd'hui que les pratiques d'infanticide ont cessé, sous l'influence des missionnaires religieux qui, pendant la période coloniale, ont joué un rôle important dans la rupture avec les pratiques ancestrales d'infanticide (Bastian, 2001). Des pratiques alternatives sont décrites, visant à ce que les enfants "extraordinaires" et leur géniteur payent une amende (par des danses et des cérémonies), les lavant de tout soupçons (Singleton, 2004). De plus, dans la plupart des pays, la mise en place d'une législation classant

Mondes en Développement Vol.37-2009/2-n¹46 
l'infanticide comme un crime lui confère un caractère illégal et puni par la loi. La création de centres d'accueil pour enfants handicapés, ou pour jumeaux, contribue fortement à limiter les pratiques d'infanticide et d'abandon en offrant une alternative acceptable aux parents. Néanmoins, les pratiques d'infanticide ont-elles totalement disparues ? Et quand ces enfants "extraordinaires" ne sont plus tués, sont-ils victimes de certaines discriminations?

Des enfants sont victimes de discrimination, qualifiée de négligence affective (Lolo, 1991), négligence sociale (Bonnet, 1997) ou d'abandon symbolique (Ezembé, 2003) de la part de leur mère ou de leur groupe social relativement à certaines croyances. Chez les Igbos du Nigeria, les pratiques de meurtre de jumeaux ne sont plus socialement valorisées. Néanmoins, des comportements de mises à distance physique (confiage d'un jumeau) ou sociale (refus d'habiller les jumeaux de manière identique, de partager leur anniversaire) sont observés (Bastian, 2001). Une étude qualitative récente montre que chez les Antambahoaka de Madagascar la pratique d'infanticide s'est transformée en une pratique d'abandon, soit par confiage dans une parenté éloignée, soit auprès de centres d'accueil en place depuis la fin des années 1980. Certains parents, choisissant d'élever leurs jumeaux, sont victimes de discrimination (Fernandes, 2008).

Il reste encore très difficile de définir précisément les pratiques discriminatoires (à l'égard des enfants ou des parents qui choisissent de ne pas respecter l'interdit) et de les quantifier, comme de s'assurer de la disparition de pratiques d'infanticide. Les études sur les pratiques d'infanticide contemporaines demeurent quasi-inexistantes. Les statistiques font cruellement défaut, et l'incomplétude de l'enregistrement des naissances à l'état civil ne permet pas d'en établir une mesure fiable. Un exemple est cependant à relever. Au Nord Ghana, le site de suivi démographique de Navrongo constitue une zone d'enquêtes fines et répétées, qui a permis une identification de ces pratiques. L'analyse des causes de décès néonatales sur une période de sept années (19952002) a montré que les décès attribués à l'infanticide représentent $5 \%$ des décès néonataux (Baiden et al., 2006). Cet indicateur, difficile à obtenir par ailleurs, serait pourtant d'un grand intérêt pour orienter les programmes d'action. Des progrès peuvent et doivent être réalisés dans l'amélioration de l'enregistrement des données nécessaires à la production de statistiques sur le devenir de ces enfants nés de circonstances "anormales". Cela passe, notamment, par l'amélioration de l'enregistrement des naissances et des décès à l'état civil.

\subsection{Les naissances conçues hors union : un autre facteur de rejet}

Les travaux de démographie historique en Europe montrent que l'une des principales causes d'abandon d'enfants entre le 17 ème et le $20^{\text {ème }}$ siècles est la stigmatisation des naissances illégitimes (Fuchs, 1992 ; Morel, 2003). Les naissances illégitimes de mères célibataires ou adultères ne trouvent aucune place au sein des familles. Elles forment l'essentiel des effectifs des centres 
d'accueil au $17^{\text {ème }}$ siècle. Au $18^{\text {ème }}$ siècle, l'Eglise met au point un système d'anonymat pour préserver la bourgeoisie de la honte des naissances illégitimes. Il s'agit des tours, compartiments pivotant à deux portes qui permet de recueillir sans délai le bébé, tout en ignorant l'identité de celui qui l'abandonne (Morel, 2003).

Cette pratique d'anonymat se retrouve en France dans les pratiques "d'accouchement sous X", fortement remises en cause par les défenseurs du droit à la connaissance des origines (Faqué, 2004 ; Guillin, 1996 ; Iacub, 2003). L'arrivée de la contraception au milieu du 20 ème siècle et la libéralisation de l'avortement en Europe ont permis l'accès à une prévention efficace des naissances non voulues et ont réduit d'autant le nombre des abandons. En France, en 1996, la proportion d'enfants abandonnés à la naissance est estimée à 1 pour 1000 (Marinopoulos, 1997).

En Afrique, hormis quelques rares exceptions, la sexualité hors mariage est encore fortement stigmatisée. Les normes sociales rejettent très souvent l'enfant né hors union, qu'il s'agisse de l'enfant adultérin ou de l'enfant du célibat, et cela de manière si forte que cette pression sociale a pour conséquence de nombreux abandons. Au Cameroun, l'accroissement des enfants abandonnés est cité comme l'un des signes les plus dramatiques de la marginalisation socio-économique des mères célibataires et de leur enfant (Calvès, 2006). On constate que les enfants recueillis dans les institutions (pouponnières, orphelinats) sont souvent abandonnés ou confiés par des mères célibataires. C'est particulièrement le cas en Algérie où une étude menée dans les années 1980 montre que les enfants abandonnés, dont le nombre est en augmentation constante, sont quasi exclusivement le fait de jeunes mères célibataires (Lacoste-Dujardin, 1986). Une étude au Sénégal analyse 33 dossiers d'expertise psychiatrique pour infanticide, entre 1968 et 1994. Seulement deux cas de pathologie mentale ont été identifiés. Il s'agit, en très grande partie, (30/33) de femmes célibataires ou mariées mais dont l'époux est parti en migration (Menick, 2000). Une autre étude de cas au Sénégal illustre l'infidélité comme cause de néonaticide (Sow et al., 1989). Une étude en milieu hospitalier à Brazzaville, présente le cas de 9 nouveau-nés hospitalisés pour prématurité ou infection néonatale en 1997 qui ont été abandonnés. Les mères étaient toutes jeunes (mineures pour les trois quarts) et sept d'entre elles célibataires (Miakayizila et al., 2000). En Tanzanie, l'analyse de 14 cas d'accusation pour infanticide par la Haute Cour de justice de Dar es Salaam, montre que les trois quarts des cas concernent des adolescentes (Rwebangira, 1994). C'est donc bien la peur du rejet familial et social qui conduit les jeunes mères célibataires et les mères adultères à l'abandon du nouveau-né, voire à l'infanticide.

\section{LES SYSTÈMES FAMILIAUX DE CIRCULATION DES ENFANTS}

L'appartenance de l'enfant au lignage plutôt qu'au couple, décrite dans de nombreuses sociétés africaines, autorise un système de don qui induit une

Mondes en Développement Vol.37-2009/2-n¹46 
circulation des enfants au sein de la parenté au sens large. Il existe en Afrique un système traditionnel de circulation des enfants, qui va du confiage temporaire et non exclusif au don exclusif d'un enfant qui se traduit par son adoption (Bledsoe, 1990 ; Castle, 1995 ; Goody, 1982 ; Jonkers, 1997 ; Lallemand, 1988, 1993 ; Madhavan, 2004 ; Rabain, 1979). Ces pratiques visent notamment à créer ou à renforcer des rapports d'entraide et des liens de parenté. Elles prennent place dans un mode d'organisation sociale et familiale conçu sur le principe d'une redistribution des charges sur l'ensemble du réseau familial (Antoine et al., 1995 ; Marie, 1997 ; Oppong, 1999 ; Pilon \& Vignikin, 2006).

C'est donc le don d'enfants qui domine les pratiques d'adoption décrites par les anthropologues, plutôt que leur abandon. Même lorsque le don d'enfant est exclusif et que le transfert des responsabilités est total, il n'y a pas de rupture avec les parents biologiques. Le don d'enfants en Afrique n'a pas pour condition d'exiger l'instauration d'une distance entre donateurs et adoptants, et encore moins d'un secret (Journet, 2004). Le don d'un enfant n'est pas honteux et ceux qui le reçoivent en sont gratifiés. Il se fait généralement quand l'enfant a atteint son autonomie et le contact avec les parents biologiques n'est pas coupé (Ezembé, 1997, 2003). En Afrique, [...] le contenu des termes 'abandon', 'adoption' est d'un sens tout autre, ne serait-ce que parce que l'enfant appartient au groupe plus qu'à ses géniteurs (Lallemand, 1988). Les transferts d'enfants sont fréquents au sein de la parenté, de manière temporaire comme définitive.

Aujourd'hui, l'urbanisation croissante et les évolutions du mode de production modifient l'organisation sociale et économique des familles, et par là, la place et le rôle de l'enfant en son sein. Différentes études mettent en évidence deux éléments : 1) les pratiques de confiage se diversifient et on voit apparaitre des situations de crise mettant l'enfant en danger (travail, exploitation physique et/ou sexuelle) ; 2) la prise en charge familiale d'enfants de la lignée, répondant à l'exigence d'une solidarité familiale révèle certaines limites face à une demande toujours plus forte et à des conditions toujours plus difficiles.

\subsection{Les nouvelles formes de circulation}

Le schéma de circulation des enfants, motivé par l'échange social, tend à se diversifier. Les transferts d'enfants sont parfois motivés aussi par des raisons économiques. Depuis plusieurs décennies, l'accès à la scolarisation est une cause de confiage d'un enfant à une famille urbaine, en échange de quelques travaux domestiques (Jonckers, 1997 ; Vandermeersch, 2002). La pauvreté rurale conduit les familles à envoyer leurs enfants chercher un emploi à la ville. Ce phénomène tend à se généraliser et, en fonction du calendrier agricole, les adolescents, de plus en plus jeunes, partent à la recherche d'un revenu complémentaire (Delaunay et al., 2006 ; Erulkar et al., 2006). Le placement d'un enfant se transforme alors en une forme de service ou de travail (Oppong, 1997). Contrairement aux transferts d'enfants harmonieux au sein de la parenté, les transferts qui se développent sous une certaine contrainte peuvent placer

Mondes en Développement Vol.37-2009/2-n¹46 
l'enfant dans un état de grande vulnérabilité, voire de danger. Ils sont exposés à plusieurs formes de discrimination (nutritionnelle, travail, violence, y compris sexuelle). L'accès au marché du travail se fait au travers de réseaux, au départ familiaux, désormais structurés et organisés, desquels le contrôle familial se trouve de plus en plus exclu. Les risques de dérives sont importants et ces aspects mériteraient d'amples investigations. Les modèles actuels de circulation des enfants s'apparentent alors à des formes perverties des modèles socialement justifiées et équilibrantes.

\subsection{Prise en charge des enfants par obligation familiale : les limites sont-elles atteintes?}

La prise en charge d'enfants se fait parfois selon certaines règles d'obligation familiale. Ainsi, un enfant devenu orphelin est généralement accueilli par ses parents les plus proches (Goody, 1982 ; Lallemand, 1993). Néanmoins, le contexte de l'épidémie de VIH/sida multiplie dans certaines régions le nombre des orphelins. Les familles en proie à la perte de leurs membres les plus productifs ont du mal à assumer la charge supplémentaire que représentent ces enfants. D'une part, les ménages doivent faire face eux-mêmes aux soins d'adultes atteints du sida et leurs revenus économiques en pâtissent. D'autre part, le niveau encore élevé de fécondité implique que les ménages doivent aussi prendre soin de plusieurs orphelins en même temps. De fait, ce système de prise en charge familial est fortement compromis par l'épidémie du sida et les besoins de soutien extérieur sont croissants (Madhavan, 2004 ; Miller et al., 2005 ; Mishra \& Bignami-Van Assche, 2008).

Par ailleurs, peu d'études se sont arrêtées sur la question des conséquences d'une naissance hors mariage sur les familles. L'accueil par une famille d'une jeune fille mère et/ou de son enfant n'est pas sans impact sur les conditions de vie de cette famille qui va devoir assumer la charge supplémentaire d'une ou de deux personnes improductives. Or, les naissances de mères célibataires sont de plus en plus nombreuses du fait du recul du mariage et de l'intensification de la sexualité juvénile (Delaunay \& Guillaume, 2007) et on peut penser que le poids de la prise en charge de ces naissances va aussi contribuer à la remise en question du système d'entraide familiale dans la prise en charge des enfants.

\section{DISCUSSION ET CONCLUSION}

L'objectif de cet article était de dresser un bilan de la connaissance de la question de l'abandon et de la prise en charge de l'enfant dans le contexte africain. Le constat général est que, si la sociologie et l'anthropologie de l'enfance apportent un cadrage qualitatif des divers modèles de prise en charge de l'enfant en Afrique, tant passés que contemporains, force est de constater que la connaissance reste très parcellaire, la classification des situations incertaine et la quantification des différents phénomènes fait défaut, tant en termes de prévalence que de tendances. Pourtant, le contexte juridique impose

Mondes en Développement Vol.37-2009/2-n¹46 
de plus en plus une prise en compte de la question de l'abandon dans les actions de protection de l'enfance. Le contexte de l'adoption internationale dans lequel s'engagent beaucoup de pays vient renforcer cette nécessité. En effet, la Convention des droits de l'enfant, proposée en 1989, a été ratifiée par l'ensemble des pays, y compris en Afrique (193 États). Face aux situations identifiées "d'enfants victimes", tels que les enfants soldats, les enfants prostitués ou objet de trafic, des protocoles facultatifs ont été proposés². Le Comité des droits de l'enfant, mis en place en 1991, est l'organe chargé de veiller à ce que les États s'acquittent des obligations qu'ils ont contractées au titre de la Convention relative aux droits de l'enfant. Chaque État doit produire un premier rapport deux ans après la ratification de la convention, puis un rapport tous les cinq ans. Ces rapports sont examinés et suivis d'observations. Le Comité des droits de l'enfant insiste sur la nécessité pour les États signataires d'organiser un suivi systématique de la réalisation des droits de l'enfant, en mettant au point des indicateurs appropriés et en recueillant des données suffisantes et fiables (Nations Unies, 2008).

Néanmoins, peu de résultats sont produits et la connaissance reste encore plus qu'approximative sur la description des situations, la mesure de leurs niveaux de prévalence, de leur tendance, de leurs causes et de leurs conséquences. Seule la multiplication des témoignages de types journalistiques ${ }^{3}$ et du développement visible des intervenants dans le domaine de la protection de l'enfance (agences internationales, $\mathrm{ONG})^{4}$, donnent une image de la diversité et de la complexité des situations rencontrées. Le vide statistique concernant la situation de la prise en charge de l'enfant est particulièrement éloquent: aucune statistique sur la prévalence, la tendance et les causes des différents types de transferts, d'abandons et de rejets d'enfants n'est disponible. Pourtant, les ruptures dans la prise en charge de l'enfant sont bien réelles. Les grandes causes de ces ruptures sont vraisemblablement encore la pauvreté et la stigmatisation sociale et culturelle de certains types de naissances (naissances "extra-ordinaires",

${ }^{2}$ Au ler février 2008, date de la clôture de la quarante-septième session du Comité des droits de l'enfant, 193 États étaient parties à la Convention relative aux droits de l'enfant. Une liste actualisée des États qui ont signé la Convention ou qui ont déposé un instrument de ratification ou d'adhésion peut être consultée sur les sites www.ohchr.org ou http://untreaty.un.org. À la même date, 119 États parties avaient ratifié le Protocole facultatif se rapportant à la Convention relative aux droits de l'enfant concernant l'implication d'enfants dans les conflits armés ou y avaient adhéré et 122 États l'avaient signé. À la même date, également, 124 États parties avaient ratifié le Protocole facultatif se rapportant à la Convention relative aux droits de l'enfant concernant la vente d'enfants, la prostitution des enfants et la pornographie mettant en scène des enfants ou y avaient adhéré et 115 États l'avaient signé. La liste actualisée des États qui ont signé les deux Protocoles facultatifs ou qui ont déposé un instrument de ratification ou d'adhésion peut être consultée sur le site www.ohchr.org (Nations Unies, 2008).

${ }^{3}$ Dans les zones urbaines, les cas de nouveaux-nés trouvés dans des lieux publics, dont la presse locale fait écho, les enfants des rues, de plus en plus visibles.

${ }^{4}$ L'augmentation du nombre des établissements d'accueil, pour la plupart issus d'initiatives religieuses ou caritatives, témoigne que des enfants se trouvent en situation d'abandon et de grande vulnérabilité.

Mondes en Développement Vol.37-2009/2-n 146 
naissances illégitimes). Face à une pauvreté toujours présente, sinon croissante, en Afrique, à l'augmentation des grossesses et des naissances non voulues (Bearinger et al., 2007 ; Bledsoe \& Cohen, 1993 ; Brown et al., 2001 ; Delaunay \& Guillaume, 2007 ; Meekers, 1994 ; Singh, 1998 ; WHO, 2007), à l'accès toujours difficile à l'avortement (Guillaume \& Molmy, 2003), à une mortalité maternelle toujours trop forte (UNICEF, 2009), on peut penser que le nombre des abandons tende à augmenter. À cette situation s'ajoute le nombre croissant des orphelins du sida et la question de leur prise en charge, qui viennent appuyer cette hypothèse. Incontestablement, la question de la prise en charge des enfants reste d'actualité, et cela dans une double perspective, à la fois celle des droits humains et celle de la santé publique.

Le développement de recherches en sciences sociales et juridiques sur ces questions apparaît donc nécessaire aux avancées politiques en matière de protection de l'enfance. Il est important d'avoir une meilleure description et compréhension des évolutions récentes. Les stratégies de prise en charge des enfants développées par les différents modèles de familles coexistants aujourd'hui (grande famille, famille nucléaire, famille monoparentale...) et les points de rupture conduisant les enfants vers des situations de grande vulnérabilité et d'exploitation doivent être explorés. Ces points de ruptures sont liés à des situations de crise conduisant à une déstructuration familiale (guerre, paupérisation, urbanisation, décès, VIH/sida). Ils le sont aussi à des phénomènes de rejet, soit de nature culturelle (croyances autour du handicap, des naissances "extra-ordinaires"), soit de nature sociale (naissances illégitimes). Pourtant, certaines données existantes peuvent être mobilisées. Les systèmes de suivi démographique et autres observatoires de population, par la finesse et la qualité d'enregistrement des événements démographiques, peuvent contribuer à identifier des pratiques d'infanticides, comme à Navrongo, Ghana (Baiden et al., 2006). En outre, ils renseignent sur le parcours de prise en charge des enfants dans les familles et donc sur le devenir des enfants socialement ou culturellement rejetés (Adjamago, Delaunay \& Mondain, 2007). De même, les grandes enquêtes, telles que les enquêtes démographiques et de santé (EDS) ou les recensements, permettent l'analyse des structures des ménages et des transferts d'enfants. De plus, une approche biographique par le recueil d'événements et d'histoires de vie peut faciliter la compréhension, à l'échelle des familles, de la succession des évènements conduisant l'enfant à sortir de la protection familiale. Enfin, d'autres données institutionnelles (archives, registres de centres d'accueil) peuvent également être mobilisées pour alimenter ces recherches.

Ces ressources qu'offrent les sciences sociales de l'enfance peuvent permettre de comprendre non seulement la dynamique des systèmes de prise en charge familiale de l'enfant et leurs points de rupture, mais aussi les systèmes de prise en charge institutionnelle qui s'organisent en réponse (État, société civile), et ainsi participer pleinement à l'élaboration des politiques publiques et aux actions de développement. 


\section{BIBLIOGRAPHIE}

ACHEBE C. (1967) Le monde s'effondre, Paris, Présence Africaine, 254 p.

ADJAMAGO A., DELAUNAY V., MONDAIN N. (2007) Les différents scénarii de la maternité prénuptiale en milieu rural sénégalais : entre déviance et tolérance, Journées scientifiques du réseau Démographie de l'AUF, Québec, AUF.

AGOSSOU T. (Ed.) (2003) Regard d'Afrique sur la maltraitance, Paris, Karthala, 277 p.

ALLOTEY P., REIDPATH D. (2001) Establishing the causes of childhood mortality in Ghana: the "spirit child", Social Science And Medicine 52 (7), 1007-1012.

ANTOINE P., BOCQUIER P., FALL A., GUISSE Y., NANITELAMIO J. (1995) Les familles Dakaroise face à la crise, Dakar, IFAN-ORSTOM-Ceped, $209 \mathrm{p}$.

BAIDEN F., HODGSON A., ADJUIK M., ADONGO P., AYAGA B., BINKA F. (2006) Trend and causes of neonatal mortality in the Kassena-Nankana district of northern Ghana, 1995-2002, Tropical Medicine And International Health, 11(4), 532-539.

BALL H., HILL C. (1996) Reevaluating Twin Infanticide, Current Anthropology, 37(5), 856-863.

BASTIAN M. (2001) The Demon Superstition : Abominable twins and mission culture in Onitsha history, Ethnology, 40(1), 13-27.

BEARINGER L. H., SIEVING R. E., FERGUSON J., SHARMA V. (2007) Adolescent health 2. Global perspectives on the sexual and reproductive health of adolescents: patterns, prevention, and potential, Lancet, 369 (9568), 1220-1231.

BLEDSOE C. (1990) The politics of children: fosterage and the social management of fertility among the Mende of Sierra Leone, In W. Handwerker (Ed.), Births and power: social change and the politics of reproduction, San Francisco, Westview Press, 81-100.

BLEDSOE C., COHEN B. (Eds.) (1993) Social dynamics of adolescent fertility in sub-Saharan Africa, Washington, National Academy Press, 208 p.

BONNET D. (1997) Autorisés à mourir ou la notion de Négligence sociale : le cas de l'enfant malnutri en Afrique de l'Ouest, Cabier de Marjuvia, 4, 43-49.

BONNET D., DE SUREMAIN C. (2008) Quelle place pour l'anthropologie de l'enfance dans le développement? Sciences au Sud, 44, 16.

BROWN A., JEJEEBHOY S., SHAHI I., TOUNT K. (2001) Sexual relations among young people in developing countries: evidence from WHO case studies, Genève, WHO.

CALVĖS A. (2006) Nouveau contexte familial à la naissance, reconnaissance paternelle et prise en charge des jeunes enfants à Yaoundé, In Aidelf (Ed.) Enfants d'aujourd'bui. Diversité des contextes, pluralité des parcours (Vol. 1), AIDELF/INED, 101-112.

CASTLE S. E. (1995) Child fostering and children's nutritional outcomes in rural Mali: the role of female status in directing child transfers, Social Science \& Medicine, 40(5), 679-693.

DELAUNAY V., ADJAMAGBO A., LALOU R. (2006) Questionner la transition de la fécondité en milieu rural africain : les apports d'une démarche longitudinale et institutionnelle, Cabiers Québécois de Démographie, 35(1), 27-50.

DELAUNAY V., GUILLAUME A. (2007) Sexualité et mode de contrôle de la fécondité chez les jeunes en Afrique sub-Saharienne, In Adjamagbo A., Msellati P., Vimard P. (Eds.), Santé de la reproduction et fécondité dans les pays du Sud. Nouveaux contextes et nouveaux comportements, Louvain-la-Neuve, Academia-Bruylant, 215-267. 
DIDUK S. (2001) Twinship and Juvenile Power: The Ordinariness of the Extraordinary. Ethnology, 40(1), 29-43.

DIDUK S. (1993) Twins, Ancestors and Socio-Economic Change in Kedjom Society. Man, 28(3), 551-571.

ERNY P. (1988) Les premiers pas dans la vie d'un enfant d'Afrique Noire, Paris, L'Harmattan, $360 \mathrm{p}$.

ERULKAR A., MEKBIB T.-A., SIMIE N., GULEMA T. (2006) Migration and Vulnerability among Adolescents in Slum Areas of Addis Ababa, Ethiopia, Journal of Youth Studies, 9(3), 361-374.

EZEMBÉ F. (2003) Don et abandon des enfants en Afrique, In Szejer M. (Ed.) Le bébé face à l'abandon, le bébé face à l'adoption, Paris, Albin Michel, 225-246.

EZEMBÉ F. (1997) Circulation des enfants en Afrique : d'hier à aujourd'hui, Journal des Psychologues, 153, 48-53.

FAQUÉ P. (2004) Né sous X: Enquête sur l'abandon, Paris, Editions Carnot, 221 p.

FERNANDES G. (2008) Twin children in Mananjary, Madagascar, Presented at 34th Biennal Congress of the International Association of Schools of Social Work Transcending Global-Local Divides, 20-24 July, Durban.

FUCHS R. (1992) Child abandonment in European History, Journal of family history, 17, 7-13.

GOODY E. (1982) Parenthood and social reproduction. Fostering and Occupational Roles in West Africa, Cambridge, Cambridge University Press, 348 p.

GUILLAUME A., MOLMY W. (2003) L'avortement en Afrique : une revue de la littérature des années 1990 à nos jours, Ceped.

GUILLIN J. (1996) De l'oubli à la mémoire : un autre regard sur l'abandon, Lyon, Editions Xavier Lejeune, $218 \mathrm{p}$.

IACUB M. (2003) Pour X. L'inconvénient d'être né de personne, Raisons Politiques, 12, 55-76.

JONCKERS D. (1997) Les enfants confiés. In Pilon M., Locoh T., Vignikin E., Vimard P. (Eds.) Ménages et familles en Afrique. Approche des dynamiques contemporaines, Paris, Ceped, 193-208.

JOURNET, N. (2004) De l'abandon au don: l'adoption dans le monde, Sciences Humaines, Hors série 45 L'enfant, 64-67.

LACHMAN, P. (2004) Understanding the current position of research in Africa as the foundation for child protection programs. Child Abuse \& Neglect, 28(8), 813-815.

LACHMAN, P. (1996) Child protection in Africa. The road ahead, Child Abuse \& Neglect, 20(7), 543-547.

LACOSTE-DUJARDIN C. (1986) Au Maghreb, l'enfance innomable. Autrement (Abandon et Adoption), 96, 85-90.

LALLEMAND S. (1993) La circulation des enfants en société traditionnelle. Prêt, don, échange, Paris, L'Harmattan, 220 p.

LALLEMAND S. (1988) Un bien qui circule beaucoup. Autrement (Abandon et Adoption), 96, 128-134.

LEVENTHAL J. (2003) The field of child maltreatment enters its fifth decade, Child Abuse and Neglect, 27(1), 1-4.

LOLO B. (1991) La dyade de la relation mère-enfant ou la prise en charge de l'enfant africain, Transitions, 31.

Mondes en Développement Vol.37-2009/2-n¹46 
MADHAVAN S. (2004) Fosterage patterns in the age of AIDS: continuity and change, Social Science \& Medicine, 58(7), 1443-1454.

MARIE A. (1997) Les structures familiales à l'épreuve de l'individualisation, In Pilon M., Locoh T., Vignikin E., Vimard P. (Eds.) Ménages et familles en Afrique. Approche des dynamiques contemporaines, Paris, Ceped, 279-300.

MARINOPOULOS S. (1997) Avant l'adoption : l'abandon, Journal des Psychologues, 153, 23-26.

MATTERN C. (2007) Etude de la pratique contumière d'infanticide et de rejet des enfants sorciers au sein de l'ethnie baribara du Nord Bénin, Université Catholique de Louvain, Louvainla-Neuve, $112 \mathrm{p}$.

MEEKERS D. (1994) Sexual initiation and premarital chilbearing in Sub-Saharan Africa, Population Studies, 48(1), 47-64.

MENICK D. M. (2000) Les contours psychosociaux de l'infanticide en Afrique noire: le cas du Sénégal, Child Abuse \& Neglect, 24(12), 1557-1565.

MIAKAYIZILA P., GANGA-ZANDZOU P., MAYANDA H. (2000) L'abandon du nouveau-né à la naissance à Brazzaville, Médecine d'Afrique Noire, 47(3), 145-148.

MILLER C., GRUSKIN S., SUBRAMANIAN S., RAJARAMAN D., HEYMANN S. (2005) Orphan care in Botswana's working households: growing responsabilities in the absence of adequate support, American Journal of Public Health, 96(8), 1429-1435.

MISHRA V., BIGNAMI-VAN ASSCHE S. (2008) Orphans and vulnerable childrenin high HIV-prevalence countries in sub-saharan Africa, DHS Analytical Studies, 15, Calverton, Maryland, Macro International Inc, $92 \mathrm{p}$.

MOREL M. (2003) Les enfants abandonnés dans la France ancienne (XVIe-XIXe siècle), In Szejer M. (Ed.) Le bébé face à l'abandon, le bébé face à l'adoption, Paris, Albin Michel, 19-45.

NATIONS UNIES (2008) Rapport du Comité des droits de l'enfant. Assemblée générale. Documents officiels. Soixante-troisième session, New York, Nations Unies.

ODEROI (2006) La violence contre les enfants dans la région de l'Océan Indien. Rapport annuel de l'Observatoire des Droits de l'Enfant dans le Région de l'Océan Indien, Maurice, ODEROI.

OPPONG C. (1999) Les systèmes familiaux et la crise économique, In Adepodju A. (ed.), La famille africaine. Politiques démographiques et développement, Paris, Karthala, 221254.

OPPONG C. (1997) African family systems and socio-economic crisis, In Adepodju A. (ed.), Family, Population and Development in Africa, London, England Zed Book, 158182.

PANTER-BRICK C., SMITH M. (ed.) (2000) Abandoned children, Cambridge University Press, 248 p.

PILON M., VIGNIKIN K. (2006) Ménages et familles en Afrique subsabarienne, Paris, Ed. des archives contemporaines/AUF, Savoirs francophones, $131 \mathrm{p}$.

RABAIN J. (1979) L'enfant du lignage. Du sevrage à la classe d'âge chez les Wolof du Sénégal, Paris, Payot, $237 \mathrm{p}$.

RAUM O. (1967) Chaga Childhood, Oxford, Oxford University Press.

RENNE E. P., BASTIAN M. L. (2001) Reviewing Twinship in Africa, Ethnology, 40 (1), $1-11$. 
RWEBANGIRA M. (1994) What has the law got to do with it?, in Tumbo-Masobo Z., Liljeström R. (ed), Chelewa, Chelewa. The dilemna of teenage girls, Östersund, The Scandinavian Institut of African Studies, 187-215.

SINGH S. (1998) Adolescent childbearing in developing countries: a global review, Studies in Family Planning, 29(2), 117-136.

SINGLETON M. (2004) Infanticide. Notes de lectures anthropologiques à usage éthique, Documents de travail du SPED, UCL Louvain-la-Neuve 20, 48.

SOW M., MOREAU J., MBAYE I., NDIAYE P., BENAIS J. (1989) Neonaticides au Sénégal : aspects sociologiques et médico-légaux, Acta Medicinae Legalis et Socialis, 39 (2), 277-283.

UNICEF (2009) La situation des enfants dans le monde 2009. La santé maternelle et néonatale, New York, UNICEF, 158 p.

UNICEF (2007a) La situation des enfants dans le monde 2008. La survie de l'enfant, New York, UNICEF, $154 \mathrm{p}$.

UNICEF (2007b) Progrès pour les enfants. Un monde digne pour les enfants : bilan statistique, New York, UNICEF, 70 p.

UNICEF (2002) Un monde digne pour les enfants, New York, UNICEF, 31 p.

VAN GENNEP A. (1904) Tabou et totétisme à Madagascar, Paris, Leroux.

VANDERMEERSCH C. (2002) Les enfants confiés âgés de moins de 6 ans au Sénégal en 1992-1993, Population, 57(4-5), 661-688.

WHO (2007) Adolescent pregnancy - Unmet needs and undoned deeds. A review of the literature and programs, Who Discussion Paper on Adolescence, $99 \mathrm{p}$. 